\title{
Friedensforschung als reflexive Wissenschaft
}

\section{Lothar Brock zum Geburtstag*}

\author{
Christoph Weller*
}

English title: Peace research as a reflexive science

\begin{abstract}
The obvious demand for more self-reflection in security policy raises the issue of reflexivity of and in peace research itself. This article recalls the reflexive potential of critical peace research, but aims to expand it to a reflexive peace research based on its claim to reconcile its scientific approach and its practice orientation. Because peace research in this view is thus constantly involved in and dealing with political conflicts, it needs a reflexive perspective to be able to scientifically analyze these conflicts. This argument is further illustrated by the methodological challenges of empirical conflict analysis. The article ends with the outlook that in addition to the critical approaches also post-colonial perspectives offer promising links to pursue peace research as a practice-oriented, reflexive science.
\end{abstract}

Keywords: Conflict analysis, critical peace research, reflexivity, post-colonial perspective

Stichwörter: Konfliktanalyse, Kritische Friedensforschung, Reflexivität, Post-koloniale Perspektive

\section{Einleitung}

„F Jührt mangelnde Selbstreflexion in der Friedens- und Sicherheitspolitik nicht zu einer verzerrten Analyse?" fragt der Call for Papers für dieses Heft der Zeitschrift „Sicherheit und Frieden“. „Doch klar!“ würden wohl die meisten antworten wollen; und das trifft leider nicht nur auf die Friedens- und Sicherheitspolitik zu, sondern etwa auch auf die Flüchtlingspolitik. Nun mag ein Beitrag der Wissenschaft zu gelingendem gesellschaftlichem Zusammenleben gerade darin bestehen, mangelnder Selbstreflexion in der Politik Einhalt zu gebieten, um ihr darüber zu vielfältigeren Einsichten zu verhelfen und größere Handlungsspielräume zu eröffnen. Dieses Prinzip liegt dem Konzept der Reflexiven Politikberatung (vgl. Weller 2003, 2017b; Daase 2006, Bröchler 2008) zugrunde. Sie leitet dazu an, die Beobachtungsweisen und traditionellen Annahmen, etwa hinsichtlich der Außen- und internationalen Politik, infrage zu stellen und zugleich - gegen die Rhetorik von der "Alternativlosigkeit" gezielt zu vervielfältigen. Aufgrund dieser konstruktivistischen Herangehensweise kann solche Politikberatung nicht behaupten, bessere Handlungsoptionen für politische Entscheidungsprozesse zu kennen, aber mehr. Und wer mehr Handlungsoptionen in Erwägung ziehen kann, ist jedenfalls in der Lage, sich möglicherweise für eine bessere zu entscheiden. ${ }^{1}$ Auf jeden Fall aber können Konsultationen entlang eines Konzepts Reflexiver Politikberatung Impulse für eine erhöhte Selbstreflexion in der Friedens- und Sicherheitspolitik leisten - vorausgesetzt die Wissenschaft leidet nicht selbst unter mangelnder Selbstreflexion. Was also könnte eine ausreichend selbstreflexive Friedensforschung auszeichnen?

* Diese Widmung soll daran erinnern, welch überragende Bedeutung Lothar Brock seit bald 50 Jahren für die deutsche Friedens- und Konfliktforschung besitzt. Schon Wilfried von Bredow (2012: 161) schrieb ihm die Rolle des „,kluge und ermutigende Einwürfe inspirierenden Präzeptors der Friedens- und Konfliktforschung“ zu, die sich nachweislich auch in diesem Aufsatz niedergeschlagen hat.

** Für ihre sehr hilfreichen Kommentare zu einer früheren Fassung dieses Textes danke ich Sabine Jaberg und Michaela Zöhrer, den Teilnehmer/innen des Forschungskolloquiums am Lehrstuhl für Politikwissenschaft, Friedens- und Konfliktforschung der Universität Augsburg sowie den zwei anonymen Gutachter/-innen der Zeitschrift "Sicherheit und Frieden".

1 Die Beurteilung, was besser sein könnte, ist nicht Teil reflexiver Politikberatung, sondern Gegenstand der politischen Auseinandersetzung in einer demokratischen Gesellschaft.
Um dieser Frage nachzugehen, möchte ich einleitend kurz an frühere Phasen der Friedensforschung und der Internationalen Beziehungen erinnern, die schon von stark reflexiven Impulsen geprägt waren und teilweise auch erkenntnistheoretische Reflexionen ihrer Forschung transparent machten (Abschnitte 2 und 3). Vor allem aber möchte ich argumentieren, dass politische Konflikte, in welche eine praxisorientierte Friedensforschung ständig involviert ist, auch Gegenstand der Friedensforschung sind. Auch die „eigenen“ Konflikte muss eine Friedensforschung wissenschaftlich analysieren können, und dies setzt voraus, reflexiv beobachten zu können (Abschnitt 4). Dabei bezieht sich diese Form der Reflexivität nicht auf eine „Soziologie der gesellschaftlichen Determinanten der soziologischen Praxis“, wie sie Bourdieu (1993: 372) vorschwebte, aber auch nicht alleine auf bestimmte, in den Sozialwissenschaften dominierende ontologische Annahmen. Das hier vertretene Verständnis von Reflexivität bezieht sich auf den Produktionsprozess wissenschaftlichen Erkennens und betrachtet die individuelle Perspektivität ebenso wie die Kommunikationsstrukturen einer wissenschaftlichen Community und die gesamte Wissenschaft als soziale Veranstaltung (vgl. Luhmann 1992; Langenohl 2009; Langer et al. 2013). Diese Reflexivität ist für die Analyse von Konflikten in einer Friedensperspektive von besonderer Bedeutung (Abschnitt 5), weshalb eine Friedensforschung stärkere Motive zu besitzen scheint als andere Disziplinen (vgl. Weller 2017a), reflexive Wissenschaft zu betreiben.

Angewandt auf den Produktionskontext dieses Beitrags (vgl. Woolgar 1988) resultiert dessen individuelle und strukturelle Perspektivität nicht nur aus meiner aktuellen Beschäftigung mit wissenschaftlicher Politikberatung auf der einen (vgl. Weller 2017b) und der Geschichte der Friedensforschung auf der anderen Seite (vgl. Bogerts et al. 2016), sondern auch aus dem Anspruch, wissenschaftliche Vorgehensweisen und Praxisorientierung als die beiden Quellen, aus denen die Friedensforschung ihre besondere Anerkennung erfährt, in einem politisierten Forschungsfeld miteinander in Einklang zu bringen. Und darin sind durchaus auch die Konsistenzbemühungen eines Wissenschaftlers erkennbar, der als ehemaliger Mitherausgeber des Friedensgutachtens (vgl. Weller et al. 2004) und langjähriges 
Vorstandsmitglied der Arbeitsgemeinschaft für Friedens- und Konfliktforschung (AFK), heute für einen politikwissenschaftlichen Lehrstuhl mit dem Schwerpunkt Friedens- und Konfliktforschung verantwortlich ist.

\section{Reflexive Impulse Kritischer Friedensforschung}

Die Kritische Friedensforschung (Vilmar 1971) besaß ein erhebliches Potenzial, reflexive Wissenschaft zu betreiben. In ihrer kritischen Gesellschaftsanalyse, einerseits bezogen auf das System organisierter Friedlosigkeit (Senghaas 1969) und andererseits auf strukturelle Gewaltverhältnisse (Galtung 1969) leistete sie wichtige Aufklärung hinsichtlich erweiterter politischer Gestaltungsmöglichkeiten des gesellschaftlichen Zusammenlebens: Indem gezeigt wurde, dass die dominanten Herrschafts- und Gewaltverhältnisse (beispielsweise das atomare Abschreckungssystem) dem Frieden nicht dienlich sind, erfuhren diese die wissenschaftliche Kritik der Friedensforschung. Und weil die derart kritisierten Strukturen in kritischen Theorieansätzen (vgl. auch Cox 1981) als menschengemacht angenommen werden, sind sie auch als historisch veränderbar zu betrachten. So verbanden sich problemlos kritische Analysen und politische Bewegungen aufgrund übereinstimmender Ziele und in Opposition zu den herrschenden Verhältnissen und Machtstrukturen, ohne allerdings dieser Perspektivität besondere - reflexive - Aufmerksamkeit zu schenken. Kritik fand nur in ontologischer Hinsicht statt, während man erkenntnistheoretisch weitgehend unkritisch blieb; die politische Brisanz der behandelten Themen schien keinen Raum zu lassen für die Reflexion der eigenen Erkenntnisbedingungen, dass diese Kritische Friedensforschung nicht außerhalb, sondern zwangsläufig in den kritisierten Strukturen stattfinden musste, und dass sie betrieben wurde von Forschenden, die in ihrem Beobachten und Analysieren, in ihren Erkenntnismöglichkeiten und in ihrem Wissenschaftsverständnis auch Kinder ihrer Zeit waren oder sind. Diese reflexive Dimension der Kritik, also die Selbstkritik bezüglich der eigenen Beobachtungsweisen und Theorieannahmen, wurde in den Ansätzen der Kritischen Friedensforschung zumeist überlagert von der Kritik an den politischen Verhältnissen und an der sogenannten „traditionellen“ (vgl. Jahn 1975: 24) oder "herkömmlichen Friedensforschung“ (vgl. etwa Senghaas 1971; Wannsee-Erklärung 1971) und deren, die herrschenden Verhältnisse zumeist stützenden Analyseergebnissen („problem-solving theories“ - Cox 1981).

In stärkerer Bezugnahme auf die Kritische Theorie der Frankfurter Schule, die auf deklamatorischer Ebene durchaus als theoretischer Hintergrund für die Kritische Friedensforschung angeführt wurde (vgl. Wasmuht 1998: 166), hätte die erkenntnistheoretische Dimension einer stark ideologiekritisch ausgerichteten Forschung (vgl. z.B. Vilmar 1971: 380ff.) zweifellos ins Blickfeld geraten können. So wie sich die Kritische Friedensforschung durch eine Abgrenzung von "traditioneller Friedensforschung“ unterscheiden wollte, hatte Max Horkheimer schon 1937 die „kritische Theorie“ als unterscheidbare Erkenntnisweise im Gegensatz zu „traditioneller Theorie“ herausgearbeitet: „Die kritische Theorie der Gesellschaft hat dagegen die Menschen als die Produzenten ihrer gesamten historischen Lebensformen zum Gegenstand. [...] Was jeweils gegeben ist, hängt nicht allein von der Natur ab, sondern auch davon, was der Mensch über sie vermag. Die Gegenstände und die Art der Wahrnehmung, die Fragestellung und der Sinn der Beantwortung zeugen von menschlicher Aktivität und dem Grad ihrer Macht" (Horkheimer 1937/1968: 57, meine Hervorhebung). Diese, im Zitat zuletzt genannte erkenntnistheoretische Problematik kritischer Wissenschaft bezogen auf die Friedensforschung zu thematisieren, blieb dann der feministischen Kritik an der Kritischen Friedensforschung vorbehalten: „wie lässt sich kritisch forschen in Anbetracht der Tatsache, daß auch kritische Friedenforschung immer schon Produkt wie auch Produzentin der zu kritisierenden unfriedlichen gesellschaftlichen Wirklichkeit ist?" (Batscheider 1993: 83). Und ganz offensichtlich war die erkenntnistheoretische Selbstkritik auch voraussetzungsreich hinsichtlich benachbarter wissenschaftlicher Diskurse, mit denen die Friedensforschung im kommunikativen Austausch stand: neben dem Feminismus auch der Konstruktivismus in den Internationalen Beziehungen.

\section{Die erkenntnistheoretische Kritik durch Feminismus und Konstruktivismus}

Die feministische Kritik an Militarismus, Krieg, Gewalt und damit verknüpften Machtstrukturen stand in enger Verbindung mit einer Kritik männlicher Wissenschaft und betraf folglich auch die Kritische Friedensforschung (vgl. Lang 1992). So kommt Batscheider (1993) in ihrer „Begründung feministischer Fragestellungen in der kritischen Friedensforschung" aufgrund ihrer erkenntnistheoretischen Reflexion, dass die Forschenden in mehrfacher Hinsicht immer auch Teil der kritisierten Gesellschaftsstrukturen sind, zu der Forderung, dass kritische Wissenschaft „für Revisionen ihres impliziten oder expliziten theoretischen Selbstverständnisses" offen sein muss (Batscheider 1993: 30): „Die selbstkritische Reflexion der Wechselwirkungen zwischen sozialem Sein und sozialwissenschaftlichem Bewußtsein ist konstitutiver Bestandteil kritischer Wissenschaft. Aus dieser Reflexion resultierende Wissenschaftskritik ist zugleich Gesellschaftskritik, wie auch Gesellschaftskritik eine Kritik der Wissenschaft impliziert" (Batscheider 1993: 30). Diese Kritik ihrer wissenschaftlichen Vorgehensweise zog sich natürlich eine Forschungsrichtung, die sich explizit als „Kritische Friedensforschung“ verstehen wollte, in besonderem Maße zu, aber sie betrifft letztlich alle sozialwissenschaftlichen Anstrengungen, welche wertorientierte Analysen der Gegenwart unternehmen und in politisierten Forschungsfeldern unterwegs sind.

Argumente für eine reflexive Wissenschaft bezogen auf Fragen von Krieg und Frieden entstanden aber nicht nur im Rahmen der Kritischen Theorie oder feministischer Ansätze, sondern auch in der Auseinandersetzung mit dem Konstruktivismus, der in den 1990er Jahren wichtige Debatten der Internationalen Beziehungen prägte. Dabei ging es zunächst um die Konzeptualisierung, Erforschung und theoretische Einbindung nicht-materieller Faktoren in politikwissenschaftliche Analyseansätze, ein für die Friedens- und Konfliktforschung schon lange Zeit durchaus vertrautes Forschungsfeld: So hatte sie bei ihrer Suche nach Ursachen von Kriegen und Gewalt sowie den Bedingungen des Friedens schon frühzeitig solchen Faktoren erhebliche Aufmerksamkeit geschenkt - ohne dabei von „Kon- 
struktionen" oder konstruktivistischen Faktoren zu sprechen: Lernpathologien (Deutsch 1963), Self-fulfilling prophecies (Kelman 1965), Feindbilder (Senghaas 1969), autistische Wahrnehmungsstrukturen (Senghaas 1972), Fehlwahrnehmungen (Jervis 1976), gesellschaftliche Projektionen (Richter 1982) oder Subjektivität (Steinweg/Wellmann 1990) sind nur einige der Stichworte und Konzepte, die in den wissenschaftlichen Analysen von Krieg und Frieden lange Zeit vor dem Aufkommen des Konstruktivismus in der politikwissenschaftlichen Teildisziplin Internationale Beziehungen eine bedeutsame Rolle spielten.

Aus diesen Einsichten über die Bedeutung subjektiver und kollektiver Konstruktionen der jeweiligen Konflikt-, Wirklichkeiten“ für das Verständnis von Konflikteskalation und die Bedingungen für Frieden und Gewaltverzicht ist jedoch in der Friedens- und Konfliktforschung nie ein systematisches konstruktivistisches Forschungsprogramm erwachsen. Zu unterschiedlich waren die jeweiligen disziplinären Hintergründe der Ansätze, zu wenig ausgeprägt die Theorieorientierung der Friedens- und Konfliktforschung und zu komplex die Wechselwirkungen zwischen den Phänomenen auf den unterschiedlichen Analyseebenen: Aus der Überwindung individueller Lernpathologien kann sich ein Wandel gesellschaftlicher Fehlwahrnehmungen ergeben, muss aber nicht, und eine im gesellschaftlichen Diskurs dominierende Konfliktwirklichkeit kann sich von jener staatlicher Entscheidungsträger/-innen erheblich unterscheiden, ohne dass erkennbar wäre, wie sich diese Differenz auf den weiteren Konfliktverlauf auswirken wird. Viele der Konzepte wurden in Einzelfallstudien entwickelt, aber nur selten in vergleichenden Analysen wieder aufgegriffen und einer theoretischen Spezifikation unterworfen. Aufgrund ihrer politischen Ausrichtung hat die Friedens- und Konfliktforschung jeweils aktuellen Themen- und Problemstellungen zumeist den Vorzug gegeben vor einer systematischen Entwicklung von Theorieansätzen mittlerer Reichweite.

Doch lieferte die Konstruktivismus-Debatte in den Internationalen Beziehungen neben zahlreichen innovativen Konzepten auch Hinweise und Anregungen zu den erkenntnistheoretischen Grundlagen einer wissenschaftlichen Erforschung von Kriegs- und Friedensursachen. Verbindendes Element dieser verschiedenen konstruktivistischen Herangehensweisen scheint Reflexion zu sein. Denn wenn es ganz allgemein gesprochen bei konstruktivistischen Analysen um „Konstruktionen“, also um (Be-)Deutungen geht, welche bestimmten Sachverhalten, Beobachtungen und Wahrnehmungen im Rahmen gesellschaftlicher Kommunikation zugewiesen werden, ist unweigerlich auch die eigene wissenschaftliche Beobachtung, Wahrnehmung und Bedeutungszuschreibung auf alle behandelten „Gegenstände“ Teil gesellschaftlicher Konstruktion der Wirklichkeit (Berger/ Luckmann 1980). Auch die Erstellung eines wissenschaftlichen Textes ist ein Konstruktionsprozess, mit dem in mehr oder weniger nachhaltiger Weise zu sozialen Konstruktionen der (politischen) Wirklichkeit beigetragen wird. Theorieaussagen über Konstruktionsprozesse aber werden zwangsläufig inkonsistent, wenn sie die in der Formulierung von Theorieaussagen „verborgenen“ Wahrnehmungen und Bedeutungszuschreibungen - die wissenschaftlichen Konstruktionsprozesse - nicht reflektieren (vgl. Weller 2005b). Und genau in diesem Sinne ist auch die wissenschaftshistorische Perspektive Teil der Reflexion des wissenschaftlichen Beobachtens einer Forschungsrichtung, also etwa der Rückblick auf Phasen und Strömungen, wie in zurückliegenden Zeiten Friedensforschung betrieben wurde (vgl. z.B. Hauswedell 1997; Jaberg 2004; Bonacker 2011; Bogerts et al. 2016).

\section{Politische Konflikte reflexiv beobachten}

Aufgrund ihres Interesses an politisch bedeutsamen Konstruktionen kommt also eine Friedensforschung nicht umhin auch zu reflektieren, welche Beiträge sie selbst durch ihre Beschreibungen und Konstruktionen zu bestimmten Weltbildern und Vorstellungen politischer Konflikte leistet. Welche Beschreibung und Analyse der aktuellen Konflikte mit Nordkorea kann politisch deeskalierend wirken und ist zugleich wissenschaftlich so informativ, dass den politischen Entscheidungsträger/-innen verschiedene Handlungsoptionen eröffnet werden, von denen wir alle nicht mit Sicherheit wissen, welche Konsequenzen sie in den kommenden Jahren zeitigen werden? Auf solche Fragen gibt es keine einfache Antwort, aber einen Modus der wissenschaftlichen Bearbeitung: Reflexivität. Wer in dieser Weise die Analyse politischer Konstruktionen (erkenntnis-)theoretisch reflektiert, kann zum einen diese Analyseergebnisse in ihrer Bedeutung für die wissenschaftliche und gesellschaftliche Konstruktion der Wirklichkeit einordnen. Zum anderen lässt sich auf diesem Wege auch ein Verständnis für die dabei eingenommene Beobachtungsposition bzw. Perspektive entwickeln. Und in dieser Reflexion lassen sich eben auch neue Einsichten über die beobachteten politischen Konflikte hervorbringen bzw. „konstruieren“ und diese dann in die politische Kommunikation einbringen.

Wenngleich eine reflexiv-konstruktivistische (vgl. Weller 2005a) Friedensforschung aus den genannten Gründen auf eine unmittelbar politische Ausrichtung und Verankerung, wie sie die Kritische Friedensforschung für sich reklamierte, verzichtet und dafür deutlich größeres Gewicht auf ihre interdisziplinäre wissenschaftliche Kommunikationsfähigkeit legt, verliert sie doch nicht ihren Bezug zur Praxis und zum politischen Diskurs. Nur stellt sie ihn - als Wissenschaft - nicht in der Weise her, dass sie bereits artikulierten politischen Positionen zu zusätzlicher gesellschaftlicher Legitimation verhilft, sondern durch systematische Reflexion des gesellschaftlichen und wissenschaftlichen Beobachtens von Konflikten. Das heißt: Im Wissen um die Muster, Einseitigkeiten und Beschränkungen des Wahrnehmens und Beobachtens und ihrer potenziell konfliktverschärfenden Wirkungen sieht eine reflexive Friedensforschung ihren Beitrag vor allem in der Analyse des Beobachtens von und in Konflikten und der Reflexion ihres jeweiligen eigenen Beobachtens.

Indem das Wissen um die Begrenztheit und Einseitigkeit jeder Erkenntnis die Möglichkeit eröffnet, in einer konflikttheoretischen Perspektive verschiedene Weltsichten nebeneinander zu betrachten und danach zu fragen, auf welchem Wege sie entstanden sind und inwieweit sie gegenseitig vermittelbar sein könnten, weist die erkenntnistheoretische Reflexion auf die Einseitigkeit und Selektivität jeder wissenschaftlichen Erkenntnis hin. Dadurch wird aber nicht nur die selbstkritische Infragestellung eigener Beobachtungs- und Theoriepräferenzen ermöglicht, sondern auch ein veränderter Umgang mit allen anderen Analysen und 
Ansätzen: Es handelt sich um unterschiedliche Perspektiven und die Gründe für die Unterschiedlichkeit lassen sich auch erkennen. Aus dieser Betrachtung aber ergeben sich verbesserte Chancen sowohl für den interdisziplinären wissenschaftlichen Austausch wie auch für den Einfluss auf politische Entscheidungen, denn die Betrachtungsweise, Begrifflichkeit und Wissensorientierung der politischen Praktiker/-innen unterscheidet sich von jener der Wissenschaft teilweise in erheblichem Maße. Die entsprechend erforderlichen Übersetzungs- und Vermittlungsleistungen werden erleichtert, wenn statt monodirektionaler akademischer Wissensvermittlung die reflexive Konsultation über die Gründe der Differenzen und Gemeinsamkeiten der Perspektiven und Weltbilder, etwa von Friedensstrategien, ermöglicht wird (vgl. Weller 2017b).

Friedenspolitische Positionen ergeben sich nicht unmittelbar aus den Resultaten der Friedensforschung, die verschiedene Perspektiven unterscheiden kann. Will sie zur Selbstreflexion der Friedens- und Sicherheitspolitik beitragen und der politischen Debatte über Frieden, Sicherheit, Krieg und Gewalt immer wieder neue Impulse verleihen, muss sie selbst reflexiv vorgehen. Dies setzt jedoch die (mehrstimmige) wissenschaftliche Beteiligung am friedenspolitischen Diskurs voraus. Dieser gesellschaftspolitischen Rolle und Verantwortung von Wissenschaft wird die Friedensforschung am ehesten gerecht, wenn sich ihre Stimmen von dem unterscheiden, was politische Akteure wie etwa die Friedensbewegung oder Menschenrechtsorganisationen in den öffentlichen Diskurs einbringen. Dies hängt auch damit zusammen, dass heute das Kennzeichen „Wissenschaft" vielleicht mehr denn je in der Gefahr steht, als Legitimationsressource für politische Überzeugungen missbraucht zu werden.

Wenn sich Friedensforschung als praxisorientierte, interdisziplinäre Wissenschaft versteht - und als solche auch wahrgenommen werden will -, kann sie auf reflexiv-konstruktivistische Perspektiven zurückgreifen. Diese stehen nicht, wie immer wieder vermutet wird, im Gegensatz zur Praxisorientierung, sondern sie sind möglicherweise erst die Voraussetzung für eine moderne, praxisorientierte Sozialwissenschaft: um nämlich im schon ausreichend vielstimmigen politisch-gesellschaftlichen Diskurs als Wissenschaft gehört zu werden.

\section{Reflexive Konfliktanalysen}

Und noch ein weiteres Argument lässt sich anführen für eine reflexive Friedensforschung, wenn diese sich konkreten gesellschaftlichen Konflikten und deren empirischer Analyse zuwenden möchte: Bei dieser wissenschaftlichen Aufgabe steht die Friedensforschung vor gleich drei Herausforderungen, die nach einer reflexiven Herangehensweise verlangen: In vielen Konflikten, denen wir uns wissenschaftlich zuwenden, finden wir uns schnell auf Seiten oder zumindest in größerer inhaltlicher Nähe zu einer der Konfliktparteien wieder - auch dann, wenn wir uns einem Neutralitätsgebot unterwürfen, denn Friedensforscher/-innen ergreifen Partei für eine zivilisierende Konfliktbearbeitung (vgl. Gulowski/Weller 2017). Das ist die erste Herausforderung, vor der wir auch in Situationen stehen, in denen wir selbst - etwa aufgrund einer überdurchschnittlichen Konfliktbereitschaft - ganz gezielt Konflikte herbeiführen und damit zwangsläufig Konfliktpartei sind. Die wissenschaftliche Analyse ist in solchen Fällen nicht unmöglich, aber sie erfordert eine Distanzierung vom eigenen Konfliktverhalten, den Rollenwechsel vom Konfliktakteur zum Analytiker bzw. zur Analytikerin, was nur durch Reflexion des eigenen Beobachtens gelingen kann: Unmittelbar während ich mich angegriffen, unterdrückt, benachteiligt oder auch nur unverstanden fühleoder mich mit den so Fühlenden solidarisiere -, aber auch während ich einen Konflikt zuspitze, in den verbalen Angriff übergehe und für meine Interessen kämpfe, kann ich weder das eigene noch das Konfliktverhalten der Gegenseite etwa daraufhin analysieren, ob es zur Eskalation oder Deeskalation der Konfliktaustragung beiträgt, ob der Konfliktgegenstand transformiert wird oder welche Institutionen der Konfliktbearbeitung einzubeziehen wären, um eine geregelte Konfliktbearbeitung zu ermöglichen (vgl. Weller 2014; Gulowski/Weller 2017). Unmittelbar anschließend lässt sich jedoch in einer reflexiven Perspektive die Selbstbeobachtung vornehmen hinsichtlich des eigenen Konfliktverhaltens, beispielsweise in welcher Rolle gegenüber dem Konflikt ich agiere (Konfliktpartei, Vermittler/ -in, Zuschauer/-in, Analytiker/-in etc.). Dadurch versetzt sich jemand dann auch in die Lage, für die nächste Konfliktphase etwa ganz bewusst einen Rollenwechsel vorzunehmen oder in einen anderen Modus der Konfliktbearbeitung zu wechseln.

Die zweite Herausforderung besteht darin, die Konsequenzen der konkreten wissenschaftlichen Konfliktanalyse für den weiteren Verlauf des analysierten Konflikts zu bedenken. Schon mit der Bezeichnung einer Uneinigkeit als „Konflikt“ findet letztlich eine Intervention in eine aktuell herausgegriffene soziale Interaktion statt, weil diese von Außenstehenden und/ oder Beteiligten dadurch anders, eben als „Konflikt“ wahrgenommen und behandelt wird. Möglicherweise haben nämlich die „Konfliktparteien“ ihre Interaktion zuvor gar nicht als „Konflikt“ wahrgenommen, sondern als Machtspiel, Abhängigkeit oder hierarchisches Ordnungsmodell. Nun aber finden sie sich aufgrund einer wissenschaftlichen Beobachtung/Deutung/ Konstruktion plötzlich in einem „Konflikt“ wieder und verhalten sich möglicherweise erst daraufhin auch entsprechend konfliktiv. So werden mit jeder weiteren analytischen Feststellung oder durch die Kommunikation mit den Konfliktparteien oder mit am Rande Beteiligten die Analysierenden auch Teil der Konfliktdynamik. Eine reflexive Friedensforschung wird (und kann) Abwägungen vornehmen, welchen eigenen Beitrag sie durch ihre Analysen zu welchem Konflikt leisten möchte und verantworten kann sowie welche Ziele sich mit der wissenschaftlichen Analyse von Konflikten erreichen lassen. Das aber setzt den oben erläuterten, gezielten Perspektivenwechsel voraus, um entsprechend unterschiedliche Weltbilder oder die Ergebnisse der Anwendung verschiedener Konflikttheorien nebeneinanderstellen und vergleichend beurteilen zu können.

Und was uns alltagspraktisch bestens vertraut ist, für die Wissenschaft aber eine erhebliche - dritte - gegenstandsspezifische Herausforderung darstellt: Über jeden Konflikt gibt es mindestens zwei „Wahrheiten“. Deshalb ist eingehend zu reflektieren, welcher Perspektive die wissenschaftliche Analyse folgen möchte. Gerade in internationalen Konflikten nehmen die beteiligten Regierungen beispielsweise erheblichen Einfluss auf die mediale Berichterstattung über den Konflikt, beschränken die Zugänge für 
unabhängige Beobachter/-innen, um gleichzeitig einen „Informationskrieg“ führen zu können. In Extremfällen kann es allein für die internationale Berichterstattung inszenierte Konfliktereignisse geben, die eine Parteinahme oder gar gewaltsame Intervention in Gang setzen sollen. Wie gehen wissenschaftliche Konfliktanalysen angesichts ihres prinzipiellen Wissens um solche Prozesse, aus denen mehrere „Konflikt-Wahrheiten“ resultieren, vor? Erst die Reflexion unseres Beobachtens (beispielsweise aufgrund welcher Annahmen wir bestimmten Quellen eher vertrauen als anderen etc.) eröffnet uns Einsichten darüber, wovon wir uns bei einer empirischen Untersuchung sozialer Konflikte angesichts der Umstrittenheit der Analysegegenstände (haben) leiten lassen, wenn wir als Friedensforscher/-in eine wissenschaftliche Konfliktanalyse vorlegen wollen.

\section{Ausblick}

Mangelnde Selbstreflexion in der Politik lässt sich - gerade in einer Demokratie als einer Herrschaftsform, die den Konfliktaustrag über politische Differenzen institutionalisiert und auf Dauer gestellt hat - mit guten Gründen kritisieren und die Friedensforschung wird im Anzetteln der entsprechenden Konflikte eine hervorgehobene Rolle einnehmen müssen. Um sich hierbei, aber auch im interdisziplinären Diskurs mit anderen Wissenschaften, die ihre eigenen Anlässe, Notwendigkeiten und Herausforderungen für erkenntnistheoretische Reflexionen hatten und haben, nicht dem Vorwurf auszusetzen, bei anderen einzuklagen, was einem selbst nicht recht gelingen mag, könnte eine reflexive Friedensforschung ein vielversprechender Weg sein, politische wie wissenschaftliche Selbstreflexion zu befördern. Nicht nur aufgrund ihres zentralen Gegenstands weltgesellschaftliche Konflikte - und ihres Interesses an politischen Konflikten drängt sich für die Friedensforschung eine reflexive Herangehensweise auf; sie kann auch auf theoretische Traditionslinien zurückblicken, an die erneut anzuknüpfen ähnlich erfolgversprechend sein könnte wie die sich intensivierende Verbindung zu de- und postkolonialen Perspektiven (vgl. Engels 2014; Brunner 2017), um auf diesen Wegen vielfältige Beiträge zum Frieden zu leisten.

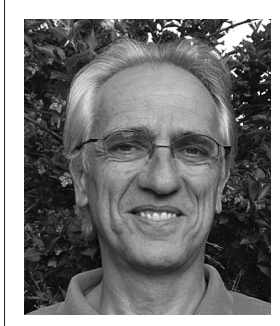

Prof. Dr. Christoph Weller ist Professor für Politikwissenschaft, Friedens- und Konfliktforschung an der Universität Augsburg, Vorstandsmitglied der Arbeitsgemeinschaft für Friedens- und Konfliktforschung (AFK) und Mitherausgeber der „Zeitschrift für Friedens- und Konfliktforschung" (ZeFKo)

\section{Literaturverzeichnis:}

Batscheider, Tordis 1993: Friedensforschung und Geschlechterverhältnis. Zur Begründung feministischer Fragestellungen in der kritischen Friedensforschung, Marburg.

Berger, Peter L. / Luckmann, Thomas 1980: Die gesellschaftliche Konstruktion der Wirklichkeit. Eine Theorie der Wissenssoziologie, 5. Auflage, Frankfurt a.M.

Bogerts, Lisa / Böschen, Stefan / Weller, Christoph 2016: Politik, Protest, Forschung - Wie entstand die Friedensforschung in der BRD? In: Wissenschaft und Frieden 1/2016, 12-15.

Bonacker, Thorsten 2011: Forschung für oder Forschung über den Frieden? Zum Selbstverständnis der Friedens- und Konfliktforschung, in: Schlotter, Peter / Wisotzki, Simone (Hrsg.): Friedens- und Konfliktforschung, Baden-Baden, 46-77.
Bourdieu, Pierre 1993: Narzißtische Reflexivität und wissenschaftliche Reflexivität, in: Berg, Eberhard / Fuchs, Martin (Hrsg.): Kultur, soziale Praxis, Text. Die Krise der ethnograpischen Repräsentation, Frankfurt a.M., 365-374.

Bredow, Wilfried von. 2012: Bestandsaufnahme, Archivpflege und Blickfelderweiterung, in: Zeitschrift für Friedens- und Konfliktforschung 1:1, 155-162.

Bröchler, Stephan 2008: Politikwissenschaftliche Politikberatung, in: Bröchler, Stephan / Schützeichel, Rainer (Hrsg.): Politikberatung, Stuttgart, 180-193.

Brunner, Claudia 2017: Friedensforschung und (De-)Kolonialität, in: Zeitschrift für Friedens- und Konfliktforschung 6: 1, 149-163.

Cox, Robert W. 1981: Social Forces, States and World Orders: Beyond International Relations Theory, in: Millennium 10: 2, 126-155.

Daase, Christopher 2006: Wissen, Nichtwissen und die Grenzen der Politikberatung Über mögliche Gefahren und wirkliche Ungewissheit in der Sicherheitspolitik, in: Hellmann, Gunther (Hrsg.): Forschung und Beratung in der Wissensgesellschaft, Baden-Baden, 189-212.

Deutsch, Karl W. 1963: The Nerves of Government: Models of Political Communication and Control, New York.

Engels, Bettina 2014: Repräsentation, Diskurse und Machtfragen. Postkoloniale Theorieansätze in der Friedens- und Konfliktforschung, in: Zeitschrift für Friedensund Konfliktforschung 3: 1, 130-150.

Galtung, Johan 1969: Violence, Peace and Peace Research, in: Journal of Peace Research 6, 167-191 (deutsch: Gewalt, Frieden und Friedensforschung, in: Senghaas, Dieter (Hrsg.): Kritische Friedensforschung, Frankfurt a.M. 1971, 55-104).

Gulowski, Rebecca / Weller, Christoph 2017: Zivile Konfliktbearbeitung: Kritik, Konzept und theoretische Fundierung, in: Peripherie 148, 386-411.

Hauswedell, Corinna 1997: Friedenswissenschaften im Kalten Krieg. Friedensforschung und friedenswissenschaftliche Initiativen in der Bundesrepublik Deutschland in den achtziger Jahren, Baden-Baden.

Horkheimer, Max 1937/1968: Traditionelle und kritische Theorie, in: ders.: Traditionelle und kritische Theorie. Vier Aufsätze, Frankfurt a.M. 1968, 12-64.

Jaberg, Sabine 2004: Realtypen der Friedensforschung. Ein deskriptiv-analytischer Versuch, in: Calließ, Jörg / Weller, Christoph (Hrsg.): Friedenstheorie: Fragen Ansätze - Möglichkeiten. 2. Auflage, Rehburg-Loccum, 49-82.

Jahn, Egbert 1975: Entwicklung und Schwerpunkte der Friedensforschung in Nordamerika und Westeuropa, in: Friedensanalysen 1, Frankfurt a.M., 15-34.

Jervis, Robert 1976: Perception and Misperception in International Politics, Princeton, N.J.

Kelman, Herbert C. (Hrsg.) 1965: International Behavior. A Social-Psychological Analysis, New York.

Lang, Susanne 1992: Ist Friedensforschung eine männliche Wissenschaft? Grundsätzliche Gedanken zu einem variationsreichen Thema, in: Wasmuht 1992, 127-139.

Langenohl, Andreas 2009: Zweimal Reflexivität in der gegenwärtigen Sozialwissenschaft: Anmerkungen zu einer nicht geführten Debatte, in: Forum Qualitative Sozialforschung 10: 2, Art. 9.

Langer, Phil C. / Kühner, Angela / Schweder, Panja (Hrsg.) 2013: Reflexive Wissensproduktion. Anregungen zu einem kritischen Methodenverständnis in qualitativer Forschung, Wiesbaden.

Luhmann, Niklas 1992: Die Wissenschaft der Gesellschaft, Frankfurt a.M.

Richter, Horst-Eberhard 1982: Zur Psychologie des Friedens, Reinbek.

Senghaas, Dieter 1969: Abschreckung und Frieden. Studien zur Kritik organisierter Friedlosigkeit, Frankfurt a.M.

Senghaas, Dieter 1971: Editorisches Vorwort, in: ders. (Hrsg.): Kritische Friedensforschung, Frankfurt a.M., 7-21.

Senghaas, Dieter 1972: Rüstung und Militarismus, Frankfurt a.M.

Steinweg, Reiner / Wellmann, Christian (Red.): Die vergessene Dimension internationaler Konflikte: Subjektivität (Friedensanalysen 24), Frankfurt a.M.

Vilmar, Fritz 1971: Systematischer Entwurf zur Kritischen Friedensforschung, in Senghaas, Dieter (Hrsg.): Kritische Friedensforschung, Frankfurt a.M., 362-395.

Wannsee-Erklärung 1971: Erklärung zur Friedensforschung, in: Senghaas, Dieter (Hrsg.): Kritische Friedensforschung, Frankfurt a.M., 416-419.

Wasmuht, Ulrike C. (Hrsg.) 1992: Ist Wissen Macht? Zur aktuellen Funktion von Friedensforschung, Baden-Baden.

Wasmuht, Ulrike C. 1998: Geschichte der deutschen Friedensforschung. Entwicklung Selbstverständnis - Politischer Kontext, Münster.

Weller, Christoph 2003: Internationale Politik und Konstruktivismus. Ein Beipackzettel, in: WeltTrends 41, 107-123.

Weller, Christoph 2005a: Gewalt, Frieden und Friedensforschung. Eine konstruktivistische Annäherung, in: Jahn, Egbert / Fischer, Sabine / Sahm, Astrid (Hrsg.): Die Zukunft des Friedens, Band 2, Wiesbaden, 91-110.

Weller, Christoph 2005b: Perspektiven eines reflexiven Konstruktivismus für die Internationalen Beziehungen, in: Ulbert, Cornelia / Weller, Christoph (Hrsg.): Konstruktivistische Analysen der internationalen Politik, Wiesbaden, 35-64.

Weller, Christoph 2014: Konfliktanalyse in der Konfliktforschung, in: Bock, Andreas M. / Henneberg, Ingo (Hrsg.): Iran, die Bombe und das Streben nach Sicherheit. Strukturierte Konfliktanalysen, Baden-Baden, 15-31.

Weller, Christoph 2017a: Friedens- und Konfliktforschung - Herausforderung für die Internationalen Beziehungen? In: Sauer, Frank / Masala, Carlo (Hrsg.): Handbuch Internationale Beziehungen, Wiesbaden, 551-572.

Weller, Christoph 2017b: Krisenprävention bedarf reflexiver Formen der Außenpolitikberatung, in: PeaceLab (http://www.peacelab2016.de/peacelab2016/, 29.5.2017).

Weller, Christoph / Ratsch, Ulrich / Mutz, Reinhard / Schoch, Bruno / Hauswedell, Corinna (Hrsg.): Friedensgutachten 2004, Münster. 